\title{
Research on Effect of CDFI on the Diagnostic Coincidence Rate of Superficial Lymph Node Tuberculosis Patients
}

\author{
Zheng Wang \\ Department of Ultrasound, Henan Province Traditional Chinese Medicine Hospital, Zhengzhou, 450002, \\ China
}

Keywords: Ultrasound Doppler Flow Imaging; Superficial Lymphadenopathy; Superficial Lymph Node Tuberculosis; Diagnostic Efficacy

\begin{abstract}
Objective: To investigate the clinical value of ultrasound Doppler flow imaging (CDFI) in the diagnosis of superficial lymph node tuberculosis (SLNT). Methods: From December 2016 to July 2018, 97 patients with superficial lymphadenopathy in our hospital were selected for CDFI. The pathological diagnosis was used as the "gold standard" to analyze the diagnostic efficacy and SLNT imaging features of CDFI. Results: In 97 patients with superficial lymphadenopathy, 36 cases of SLNT and 61 cases of non-SLNT were diagnosed by pathological diagnosis. 37 cases of SLNT and 60 cases of non-SLNT were detected by CDFI. The specificity of CDFI diagnosis was $91.80 \%$ (56/61). For $88.89 \%$ (32/36), the positive predictive value was $86.49 \%(32 / 37)$, the negative predictive value was $93.33 \%$ (56/60), the diagnostic coincidence rate was 0.907 , the Kappa index was 0.802, and the 95\% CI was 0.603 1.001, $\mathrm{P}=0.000$; patients with SLNT had higher PSV and RI than patients with benign lesions $(\mathrm{P}<0.05)$; the prone sites showed bilateral or one-sided hypoechoic nodules, and enlarged lymph nodes often became round, with the progress of the disease, internal echo weakened, the lymphatic structure was completely destroyed in the late stage, the echo was disordered, the CDFI explored and the blood flow in the lesion was rare, the peripheral blood flow was rich, and there was no blood flow signal inside the abscess. Conclusion: The PSV and RI of patients with SLNT are significantly increased. The CDFI diagnosis has specific imaging findings, which can provide data support for clinical diagnosis and treatment planning.
\end{abstract}

\section{Introduction}

Superficial lymph node tuberculosis (SLNT) is a tumor in which the body specializes in anti-virus and disinfection, and the lymphatic system that protects blood vessels is concentrated and condensed on the muscle surface tissue when it is attacked by germs that are difficult to kill in vitro and in vivo. The disease is more than the neck on one side or one side of the neck, no pain, no itching, push and slide, if the body's resistance is poor, it will gradually increase, and even repeated ulceration, secondary systemic poisoning symptoms [1]. In the long run, the diagnosis of SLNT is mainly based on clinical features, history of tuberculosis infection, pathological tissue biopsy, etc. In recent years, with the improvement of ultrasound diagnostic system and the improvement of technology, it has been widely used in the diagnosis of various diseases [2]. This study selected 97 patients with superficial lymphadenopathy in our hospital for the purpose of analyzing the clinical diagnostic value of ultrasound Doppler flow imaging (CDFI). The report is as follows.

\section{Materials and Methods}

General data: From December 2016 to July 2018, 97 patients with superficial lymphadenopathy in our hospital were selected, including 41 males and 56 females, aged 29-54 years, mean (41.45 \pm 6.05 ) years old. The body mass index was $18.1 \sim 23.6 \mathrm{~kg} / \mathrm{m} 2$, with an average of $(20.86 \pm 1.22) \mathrm{kg} / \mathrm{m} 2$, including 15 cases of axillary lymph node enlargement, 65 cases of cervical lymphadenopathy, 12 cases of inguinal lymphadenopathy, and supraclavicular lymphadenopathy. This study meets the relevant requirements of the Helsinki Declaration of the World Medical Association. 
Inclusion and exclusion criteria (1) Inclusion criteria: all diagnosed by pathological diagnosis; both informed and signed the consent form. (2) Exclusion criteria: those with mental disorders; those with incomplete clinical data.

Methods (1) Both CDFI detection, ultrasound diagnosis system (Qinhuangdao Kangtai Medical System Co., Ltd., KAI-X3 type), probe frequency $6 \sim 10 \mathrm{MHz}$, comprehensively scan the lesion area, first observe the size of the lesion by two-dimensional ultrasound, package Membrane, morphology, internal echo, relationship with nearby tissues, etc., and then observe the distribution of internal and peripheral blood flow by CDFI, including blood flow, blood flow morphology and number. Wall filter is selected as low gear, the color gain is appropriately increased, and no pressure is applied. Pulsed Doppler sampling is performed on the internal blood flow with the aid of CDFI, and the arterial resistance index (RI) and the highest frequency spectrum (PSV) is recorded. Tuberculous lymph nodes are seen in the cortex and medulla, and they are mixed blood flow, recorded $(+)$, otherwise (-). (2) Pathological diagnosis: The sections $(5-7 \mu \mathrm{m})$ were examined by HE staining microscopy; the fine needles were taken up by cytology smear HE staining microscopy, and the magnification was 100-400 times. The structure of the lymph nodes is disordered, and the follicles disappear. It can be seen that tuberculous granuloma and caseous necrosis are tuberculous lymph nodes (+), otherwise (-).

Observation indicators. (1) Analysis of pathological diagnosis and CDFI test results. (2) Compare PSV and RI in patients with SLNT and benign lesions. (3) Analysis of CDFI diagnostic efficacy. (4) Analysis of SLNT imaging features.

Statistical analysis. The data were processed by SPSS21.0. The measurement data (PSV, RI) were expressed as $( \pm \mathrm{s})$, and the t-test was performed. $\mathrm{P}<0.05$ was considered statistically significant.

\section{Results}

Test results 97 cases of superficial lymphadenopathy were diagnosed by pathological diagnosis of 36 cases of SLNT, 61 cases of non-SLNT; 37 cases of SLNT were detected by CDFI, 60 cases of non-SLNT, see Table 1.

Table 1 Test Results

\begin{tabular}{llll}
\hline $\begin{array}{l}\text { Pathological } \\
\text { diagnosis }\end{array}$ & CDFI diagnosis & Total \\
\cline { 2 - 3 } & + & - & 36 \\
+ & 32 & 4 & 61 \\
- & 5 & 56 & 97 \\
\hline
\end{tabular}

Blood flow The PSV and RI of SLNT patients were higher than those of benign lesions $(\mathrm{P}<0.05)$, see Table 2.

Table 2 blood flow conditions ( \pm s)

\begin{tabular}{llll}
\hline Category & Number of cases & PSV $(\mathrm{cm} / \mathrm{s})$ & RI \\
\hline Benign lesion & 61 & $17.98 \pm 5.66$ & $0.63 \pm 0.07$ \\
SLNT & 36 & $21.98 \pm 6.14$ & $0.71 \pm 0.06$ \\
$t$ & & 3.258 & 5.725 \\
$P$ & & 0.002 & 0.000 \\
\hline
\end{tabular}

Diagnostic efficacy. CDFI diagnostic specificity was $91.80 \%$ (56/61), sensitivity was $88.89 \%$ (32/36), positive predictive value was $86.49 \%$ (32/37), negative predictive value was 93.33\% (56/60), The diagnostic coincidence rate was 0.907, the Kappa index was 0.802, and the 95\% CI was 0.603 to $1.001, \mathrm{P}=0.000$.

SLNT imaging findings (1) See the bilateral or one-sided hypoechoic nodules in the prone site, and many strings are arranged along the large blood vessels. (2) The enlarged lymph nodes are often 
round, the early capsule is intact, the endothelium boundary and the boundary are still clear, and some lymph node squeezing disappears or moves to the periphery. (3) As the disease progresses, the internal echo are weakened, the nodules are in harmony, and the boundary is blurred. (4) The lymphatic structure is completely destroyed in the late stage, the echo is disorderly, and the shape is irregular and changeable; some see strong echoes, light spots, and sound shadows at the rear, which are calcifications of tuberculosis. (5) Part of the nodules of tuberculosis rupture, see subcutaneous extension of the tubular hypoechoic, is the formation of sinus. (6) CDFI probes and the blood flow in the lesion is rare, the peripheral blood flow is rich, and there is no blood flow signal inside the abscess.

\section{Discussion}

The lymphatic system is an important component of the vascular system of the body. Any antigenic stimulation induces lymphatic response and secondary lymph node hyperplasia [3]. Superficial lymph node enlargement induces a variety of factors, in addition to lymph node tuberculosis, common malignant lymphoma, lymphadenitis, etc., the pathology is different, the treatment is different [4-5]. Therefore, clinical attention should be paid to differential diagnosis.

The normal lymph node sonogram is mostly round or elliptical, with one side concave. The other side of the protuberance, called the door, belongs to the site of neurovascular access [6]. A large number of clinical studies have indicated that most of the lymphographic images correspond to their structures, such as the narrower high echo zone of the central genus, surrounded by low echo, and in most cases CDFI can detect linear or scintillation-like blood in the medulla or cortex. Flow signals, a few large lymph nodes see increased blood flow signals, showing a regular distribution [7-8]. According to the disease process and symptoms, SLNT is divided into the initial stage, the stage of emery and pus, the initial skin color is unchanged, and one or several nodules are pushed, which can be moved, and it is firm, not painful or hot, and internal medulla. The pressure disappeared or squeezed to the periphery; in the middle stage, as the disease progressed, the skin color of the lymphatic tuberculosis area became dark red, and it did not move. The ultrasound showed an increase in the size of the lymph nodes, and some of the mutual fusion became a string, the boundary was not clear, and the internal echo was weakened. In the late lesions, there are lymph nodes, gradually pain, abscesses are broken, ultrasound shows lymphatic structure destruction, irregular shape, and echoes [9]. The SLNT imaging findings of this study are consistent with the above findings.

In addition, SLNT needs to be differentiated from other superficial lymphadenopathy. The lymph node metastasis is nearly circular, with a long-to-short diameter ratio of $<2$, the border is rough, the internal hypoechoic, the light spot is thick, and the CDFI shows the center and surrounding blood flow signals are disordered. Rich, no blood flow signal in the necrotic area; lymphadenitis is mostly elliptical, the cortex and medulla grows evenly, the boundary is smooth, and the echo of chronic lymphadenitis is enhanced and thickened obviously. CDFI shows that the blood flow is distributed along the medulla. This study showed that the PSV and RI of SLNT patients were significantly higher than those of benign lesions $(\mathrm{P}<0.05)$, suggesting that the clinical diagnosis of SLNT can be further differentiated by measuring PSV and RI. In this study, 97 patients with superficial lymphadenopathy were diagnosed by pathological diagnosis of 36 cases of SLNT. 37 cases of SLNT were detected by CDFI. The specificity was $91.80 \%$, the sensitivity was $88.89 \%$, and the positive predictive value was $86.49 \%$. The negative predictive value was negative predictive value. 93.33\%, the diagnostic coincidence rate is 0.907 , indicating that CDFI has a high detection rate of SLNT and has certain diagnostic value, which can provide data support for clinical diagnosis and treatment.

In summary, patients with SLNT have a significant increase in PSV and RI, and have specific imaging findings using CDFI diagnosis, which can provide data support for clinical diagnosis and treatment planning. 


\section{References}

[1] Liang Jianying, Zhang Changyan, He Ronghui. Effect of anti-tuberculosis treatment on CD4+ lymphocytes and prognosis in patients with acquired immunodeficiency syndrome and pulmonary tuberculosis[J].Clinical Misdiagnosis and Mistreatment,2018,31(02):66- 69.

[2] Wang Zeai, Ding Yongning, Fan Yunqing. Diagnostic value of ultrasound elastography combined with CDFI in axillary lymph nodes of breast cancer patients[J].Journal of Xuzhou Medical College,2016,36(11):770-773.

[3] Peng Wei, Wei Songfeng, Zheng Xiangqian, et al. Clinicopathological features of 1401 cases of thyroid micropapillary carcinoma and risk factors of central lymph node metastasis[J]. Chinese Journal of Clinical Oncology, 2016, 43(3): 95-99.

[4] Niu Jianming, Tan Yanhui, Zhang Dan, et al. Clinical efficacy of local injection combined with oral rehabilitation new fluid in the treatment of superficial lymph node tuberculosis[J].Guangxi Medical Journal,2016,38(2):187-188.

[5] Xu Junli, Cui Yuanbo, Chen Qiliang, et al. Application of anatomy and protection of cervical sheath and important nerve in cervical lymph node dissection in cervical lymph node tuberculosis[J]. Laboratory Medicine and Clinical Medicine, 2018, 15(06):782-784,788.

[6] Hua Qifeng, Hu Airong, Zhang Yena, et al. Diagnostic value and imaging findings of CT applied to patients with acquired immunodeficiency syndrome with pulmonary tuberculosis infection [J]. Chinese Journal of Nosocomiology, 2017, 27(10): 2241 -2244.

[7] Cui Qiuli, Yin Shanshan, Fan Zhihui, et al. Ultrasound contrast enhancement mode and temporal-intensity curve morphological features of superficial lymph nodes [J]. Chinese Journal of Ultrasound Imaging, 2016, 25(12): 1055-1059.

[8] Sun Wenwen, Wu Furong, Xiao Heping, et al. Early diagnostic value of ultrasound bronchoscopy biopsy for adult mediastinal lymph node tuberculosis (a report of 87 cases) [J]. China National Defense Magazine, 2014, 36 (3): 180-183.

[9] Meng Jun, Yang Gaoyi, Wen Boping, et al. Ultrasound characteristics of calcification in cervical tuberculous lymph nodes and metastatic lymph nodes of papillary thyroid carcinoma[J]. Chinese Journal of Ultrasound in Medicine, 2015, 31(6): 487 -489.

[10] Wang Xiaorong, Ai Dubai, Mu Hemai, Zhang Rong, et al. Three-dimensional ultrasound combined with contrast-enhanced ultrasonography to observe the characteristics of atypical lymph nodes in the neck[J]. Chinese Journal of Medical Imaging Technology, 2016, 32(8): 1195- 1199. 